University of Wollongong

Research Online

Faculty of Engineering - Papers (Archive)

Faculty of Engineering and Information

Sciences

$1-1-2005$

\title{
Terahertz absorption by magnetoplasma sound wave excitation in
} semiconductor heterostructures

\author{
Chao Zhang \\ University of Wollongong, czhang@uow.edu.au \\ S. Hessami Pilehrood \\ University of Wollongong \\ Roger A. Lewis \\ University of Wollongong, roger@uow.edu.au
}

Follow this and additional works at: https://ro.uow.edu.au/engpapers

Part of the Engineering Commons

https://ro.uow.edu.au/engpapers/4120

\section{Recommended Citation}

Zhang, Chao; Pilehrood, S. Hessami; and Lewis, Roger A.: Terahertz absorption by magnetoplasma sound wave excitation in semiconductor heterostructures 2005, 69-72.

https://ro.uow.edu.au/engpapers/4120

Research Online is the open access institutional repository for the University of Wollongong. For further information contact the UOW Library: research-pubs@uow.edu.au 


\title{
Terahertz absorption by magnetoplasma sound wave excitation in semiconductor heterostructures
}

\author{
C. Zhang, S. Hessami Pilehrood, and R. A. Lewis \\ School of Engineering Physics and \\ Institute of Superconducting and Electronic Materials, \\ University of Wollongong, Wollongong NSW 2522, Australia \\ Email: czhang@uow.edu.au
}

\begin{abstract}
By employing the exact time-dependent wavefunctions for an electron gas under a quantizing magnetic field and laser radiation, we study the dielectric properties of a system when the laser frequency equals the cyclotron frequency. This resonant condition leads to a new magneto-photon-plasmon (magnetopolariton) mode. Unlike the ordinary magnetoplasmon, the energy of the new mode increases linearly with the radiation intensity. As the wavenumber increases, this new mode behaves like a sound wave. The absorption due to the plasmon mode, $\Im m[1 / \epsilon(q, \omega)]$, is presented.
\end{abstract}

\section{INTRODUCTION}

The experimental observation of magnetoresistance oscillations (MRO) and zero-resistance states (ZRS) in high-mobility two-dimensional electronic systems [1]-[3] has stimulated renewed interest in the transport properties of electronic systems under electromagnetic (EM) radiation [4]-[7]. Both MRO and ZRS are the consequence of the interplay of the photon frequency and the cyclotron frequency. Due to the resonant scattering of electrons by impurities under EM radiation, electrons can travel through different orbits without loss of energy. Apart from MRO and ZRS, other interesting radiationinduced phenomena in electronic systems have been reported in recent years, including: impact ionization under terahertz radiation [8], nonlinear transport [9]-[11], resonant absorption [12], the photon enhanced hot-electron effect [13], the LO-phonon bottleneck effect [14], and THz-photon-assisted tunneling [15], [16].

In this paper, we report another interesting phenomenon in a magnetically-quantized electronic system under EM radiation. It has long been recognized that the dielectric function of an electron gas under a quantizing magnetic field plays an important role in the understanding of optical and transport properties at low temperature, for example, the magnetoexciton effect and the quantum Hall effect. The absorption of the radiation field can be studied through the knowledge of the structural factor, $\frac{\hbar}{2 \pi e^{2}} \Im(1 \epsilon(\omega))$, also known as the structural function. The poles in $1 \epsilon(\omega)$ are of particular importance as they determine the spectrum of collective excitations, such as magnetoplasmons and rotons. The structural factor consists of two parts,

$$
(\mathbf{q} \omega)=\frac{-}{2 \pi^{2}} \epsilon_{I}(\omega)|\epsilon(\omega)|^{2}+\frac{-}{2 \pi^{2}} \pi \delta\left(\epsilon_{R}(\omega)\right)
$$

In the absence of exchange and correlation, and disorderinduced finite-life-time effects, only collective excitations contribute to the radiation energy loss because the first term in Eq. (1) vanishes in the lowest order. The properties of magnetoplasmon oscillations in different systems and using different models have been studied. In all these models, the plasma frequency, $\omega_{p}(\quad)$ goes to zero with and. In what follows we shall show that if the system is strongly coupled to a radiation field whose frequency is equal to the cyclotron frequency, the collective excitation energy of the system actually increases rapidly with decreasing field in the small- , strong- regime. In the large- regime, the new mode behaves like a sound wave, with the speed of the plasma wave being much higher than the Fermi velocity of the system at a moderate electric field.

Consider a two-dimensional electron in the $x-y$ plane. We choose the direction of the quantizing magnetic field to be the $\mathrm{z}$-direction, $\mathbf{B}=\mathbf{e}_{\mathbf{z}}$, and the laser field to be along the y-direction, $\mathbf{E}()=\sin \left(\omega_{\gamma}\right) \mathbf{e}_{\mathbf{y}}$, where and $\omega_{\gamma}$ are the amplitude and frequency of the laser field. In the absence of the laser field, the Schrödinger equation for a single electron is

$$
-{ }_{-} \psi(\mathbf{r} \quad)=\frac{\left(\mathbf{p}-\mathbf{A}_{\mathbf{1}}\right)^{2}}{2 *} \psi(\mathbf{r})
$$

Here $*$ is the effective mass of the electron. If the Landau gauge is used, $\mathbf{A}_{1}=\mathbf{e}_{\mathbf{y}}$, and the wavefunction is given in terms of Landau states,

$$
\psi(\mathbf{r})=\exp \left(-\epsilon_{n}{ }^{-}\right) \exp \left(y_{y}\right) \phi_{n}\left(-o^{\circ}\right)
$$

where $\phi_{n}(-o)$ is the solution of a linear harmonic oscillator, ${ }_{o}=y^{2}$ is the center coordinate and $=\sqrt{-}$ is the magnetic length, $\epsilon_{n}=(+12)^{-} \omega_{c}$ are the Landau levels, and $\omega_{c}=\quad *$ is the cyclotron frequency.

In the presence of an intense laser radiation, the system is strongly coupled to the photon field, and the interplay of the magnetic field and the laser can cause strong transition between various electronic states. Let us choose the vector potential for the laser field to be in the form $\mathbf{A}_{2}=$ ( $\omega) \cos (\omega) \mathbf{e}_{\mathbf{y}}$. For notational convenience, we set ${ }^{-}=$ 1. The time-dependent wavefunction can then be obtained in terms of these unitary transformations,

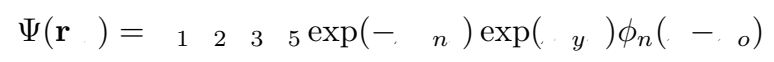


The unitary transformations can be derived analytically, $1=\exp \left[{ }_{1}()\right], \quad 2=\exp [, \quad 2()], \quad 3=\exp \left[\begin{array}{ll}x & 3\end{array}()\right]$ and ${ }_{5}=\exp \left[y_{5}()\right]$ with the initial condition ${ }_{i}(=$ $0)=1$ The solutions are,

$$
\begin{gathered}
1()=\frac{2}{2 *}\left(\frac{1}{\omega_{c}^{2}-\omega_{\gamma}^{2}}\right)^{2} \times \\
{\left[\frac{\omega_{c}^{3}}{2 \omega_{\gamma}^{2}} \sin \left(2 \omega_{c}\right)+\frac{\omega_{\gamma}}{4}\left(\frac{3 \omega_{c}^{2}}{\omega_{\gamma}^{2}}-1\right) \sin \left(2 \omega_{\gamma}\right)+\right.} \\
\left.+\left(\frac{\omega_{c}^{2}-\omega_{\gamma}^{2}}{2}\right)-\frac{\omega_{c}^{3}}{\omega_{\gamma}^{2}}\left\{\sin \left[\left(\omega_{c}-\omega_{\gamma}\right)\right]+\sin \left[\left(\omega_{c}+\omega_{\gamma}\right)\right]\right\}\right] \\
{ }_{2}()=\left(\frac{\omega_{c}}{\omega_{c}^{2}-\omega_{\gamma}^{2}}\right)\left[\frac{-\omega_{c}}{\omega_{\gamma}} \sin \left(\omega_{c}\right)+\sin \left(\omega_{\gamma}\right)\right] \\
{ }_{5}()=\frac{\omega_{c}}{\omega_{\gamma}}\left(\frac{1}{\omega_{c}^{2}-\omega_{\gamma}^{2}}\right)\left[-\cos \left(\omega_{c}\right)+\cos \left(\omega_{\gamma}\right)\right] \\
\left(\frac{1}{\omega_{c}^{2}-\omega_{\gamma}^{2}}\right)\left[\frac{\omega_{c}}{\omega_{\gamma}} \sin \left(\omega_{c}\right)-\sin \left(\omega_{\gamma}\right)\right]
\end{gathered}
$$

In the coordinate representation, ${ }_{3}$ behaves like a translational transformation and therefore its effect is to change the variable in $\phi$ to $+{ }_{3}()$. The time-dependent wavefunction is now written as

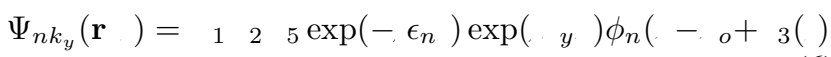

This situation can be viewed as that the center coordinate is now time-dependent and oscillates with the laser field. At resonance, $\omega_{\gamma}=\omega_{c}$ we have,

$$
\begin{gathered}
{ }_{2}()=\omega_{c} \\
{ }_{3}()={\overline{2}^{*}}^{2} \\
{ }_{5}()={\overline{\omega_{c}}}^{*}
\end{gathered}
$$

We now define the one-particle Green's function in terms of $\Psi$,

$$
\begin{gathered}
{ }^{0}\left(\mathbf{r} \mathbf{r}^{\prime} ; \quad{ }^{\prime}\right)=-\sum_{n k_{y}} \Psi_{n k_{y}}(\mathbf{r}) \Psi_{n k_{y}}^{*}\left(\mathbf{r}^{\prime} \quad '\right) \times \\
{\left[\left(1-{ }_{n, k_{y}}\right) \theta\left(-{ }^{\prime}\right)-{ }_{n, k_{y}} \theta\left({ }^{\prime}-\right)\right]} \\
\sum_{n k_{y}} i\left(u_{1}(t)-u_{1}\left(t^{\prime}\right)\right) i\left(x u_{2}(t)-x^{\prime} u_{2}\left(t^{\prime}\right)\right)-i \epsilon_{n}\left(t-t^{\prime}\right) / \hbar \times \\
i k_{y} \frac{e E}{\omega_{c} m^{*}}\left(t-t^{\prime}\right) i k_{y}\left(y-y^{\prime}\right) \times \\
\phi_{n}\left(-{ }_{o}+{ }_{3}()\right) \phi_{n}\left({ }^{\prime}-{ }_{o}+{ }_{3}\left({ }^{\prime}\right)\right) \times \\
{\left[\left(1-{ }_{n, k_{y}}\right) \theta\left(-{ }^{\prime}\right)-{ }_{n, k_{y}} \theta\left({ }^{\prime}-\right)\right]}
\end{gathered}
$$

where ${ }_{n, k_{y}}=\left[\exp \left(\epsilon_{n}+\gamma^{-}\right)+1\right]^{-1}$ is the Fermi-Dirac distribution function with energies shifted by an amount of $\gamma=\begin{array}{lllll}2 & 2 & 2\end{array}{ }^{*} \omega_{c}^{2}$ which is also known as the dynamic
Franz-Keldysh shift. $\theta($ ) is a unit step function. We now perform spatial Fourier transformations to obtain,

$$
\left.{ }^{0}\left(\mathbf{q} \mathbf{q}^{\prime} ; \quad{ }^{\prime}\right)=\int \begin{array}{lllll}
\mathbf{r} & \mathbf{r}^{\prime} i \mathbf{q} \cdot \mathbf{r}-i \mathbf{q}^{\prime} \cdot \mathbf{r}^{\prime} & 0 & \left(\mathbf{r} \mathbf{r}^{\prime} ; \quad\right. & \prime
\end{array}\right)
$$

Integrating over, , ' and $y$ results in $y=y_{y}^{\prime}$ and ${ }^{0}\left(\mathbf{q} \mathbf{q}^{\prime} ; \quad '\right)$ can now be written as

$$
\begin{gathered}
0\left(\mathbf{q} \mathbf{q}^{\prime} ; \quad,\right)=\sum_{n} \int \quad, \quad \widehat{i q_{x} x}-i \widehat{q_{x}^{\prime} x^{\prime}} \times \\
\phi_{n}\left(-{ }_{o}-{ }_{3}()\right) \phi_{n}\left({ }^{\prime}-{ }_{o}-{ }_{3}\left({ }^{\prime}\right)\right)^{-i \epsilon_{n}\left(t-t^{\prime}\right) / \hbar} \times \\
i q_{y} \frac{e E}{\omega_{c} m^{*}}\left(t-t^{\prime}\right)\left[\left(1-{ }_{n, q_{y}}\right) \theta\left(-{ }^{\prime}\right)-{ }_{n, q_{y}} \theta\left(^{\prime}-\right)\right]
\end{gathered}
$$

where $\widehat{{ }_{x}}={ }_{x}+{ }_{2}()$ and $\widehat{{ }^{\prime}}{ }_{x}={ }_{x}+{ }_{2}\left({ }^{\prime}\right)$. Introducing the center of mass and relative coordinates $=\left(+{ }^{\prime}\right) 2$, $r=-{ }^{\prime}, \quad=\left(\widehat{c}_{x}+\widehat{ }^{\prime}{ }_{x}\right) 2$ and ${ }_{r}=\widehat{ }_{x}-\widehat{'}_{x}$, and carrying out the -integration, we obtain

$$
\begin{aligned}
& =-i Q\left(u_{3}(t)-u_{3}\left(t^{\prime}\right)\right) i q_{r}\left(2 x_{o}-u_{3}(t)-u_{3}\left(t^{\prime}\right)\right)-\left(q_{r} l / 2\right)^{2} \times \\
& \int \quad i \sqrt{2} Q l u-u^{2} / 2 n\left[2+\frac{(r)^{2}}{2}\right]
\end{aligned}
$$

where $=\left(r+{ }_{3}()-{ }_{3}\left({ }^{\prime}\right)\right)(\sqrt{2})$.

The electronic polarization function $\Pi\left(\mathbf{q} \mathbf{q}^{\prime} ; \quad\right.$ ') is given by

$\Pi\left(\mathbf{q} \mathbf{q}^{\prime} ; \quad{ }^{\prime}\right)=-2 \sum_{n k_{y}} \sum_{n^{\prime} k_{y}^{\prime}} \int \mathbf{r} \Psi_{n^{\prime} k_{y}^{\prime}}^{*}(\mathbf{r} \quad)^{i \mathbf{q} \cdot \mathbf{r}} \Psi_{n k_{y}}(\mathbf{r} \quad) \times$

$$
\int \mathbf{r}^{\prime} \Psi_{n k_{y}}^{*}\left(\mathbf{r}^{\prime} \quad{ }^{\prime}\right)^{-i \mathbf{q}^{\prime} \cdot \mathbf{r}^{\prime}} \Psi_{n^{\prime} k_{y}^{\prime}}\left(\mathbf{r}^{\prime} \quad{ }^{\prime}\right)\left[n_{n, k_{y}}-{ }_{n^{\prime}, k_{y}+q_{y}}\right]
$$

Integrating over results in ${ }_{y}^{\prime}={ }_{y}+{ }_{y}$ and $y={ }_{y}^{\prime}$. The polarization function can be further written as

$$
\begin{aligned}
& \Pi\left(\mathbf{q} \mathbf{q}^{\prime} ; \quad '\right)=-2 \sum_{n k_{y}} \sum_{n^{\prime}} \times \\
& n n^{\prime}(x \quad y \quad y+y ;) \quad n n^{\prime}\left(-{ }_{x}^{\prime} \cdot y \cdot y-y ;{ }^{\prime}\right) \times \\
& \exp \left\{-\frac{x}{2^{*}}\left({ }^{2}-{ }^{\prime 2}\right)\right\} \exp \left\{-\frac{y}{\omega_{c}{ }^{*}}\left(-{ }^{\prime}\right)\right\} \times \\
& {\left[n, k_{y}-n^{\prime}, k_{y}+q_{y}\right]^{-i\left(\epsilon_{n}-\epsilon_{n^{\prime}}\right)\left(t-t^{\prime}\right)}}
\end{aligned}
$$

where

$$
\begin{aligned}
& n n^{\prime}(x \quad y \quad y+y)=
\end{aligned}
$$

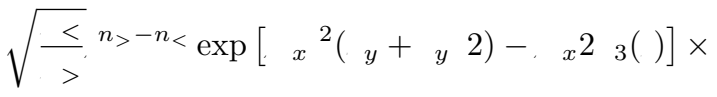

$$
\begin{aligned}
& \exp (-\quad 2) \quad\left(n_{>}-n_{<}\right) / 2 \quad \begin{array}{l}
n_{>}-n_{<}(\quad) \\
n_{<}
\end{array} \\
& =\exp \left[\begin{array}{lll}
x^{2} & y
\end{array}\right] \quad n n^{\prime}\left(\begin{array}{ll}
x & y
\end{array}\right)
\end{aligned}
$$

where $=\left(\begin{array}{ll}1 & 2\end{array}\right)()^{2}$. Substituting (16) in (15) and summing over $y$ gives ${ }_{x}^{\prime}={ }_{x}$. The result that $\mathbf{q}=\mathbf{q}^{\prime}$ shows that system's translational invariance is not affected by a perpendicular 
magnetic field and long-wavelength electromagnetic radiation. After introducing $\tau=-{ }^{\prime}$ and $=\left(+{ }^{\prime}\right) 2$, we obtain

$$
\begin{gathered}
\Pi(\mathbf{q} ; \tau)=-2 \sum_{n n^{\prime}} n n^{\prime}\left(\begin{array}{ll}
x & y
\end{array}\right) n n^{\prime}\left(\begin{array}{cc}
x & y
\end{array}\right) \times \\
\exp \left\{-\frac{x}{2 *} \tau\right\} \exp \left\{-\frac{y}{\omega_{c} *} \tau\right\}\left[\begin{array}{ll}
n & -n^{\prime}
\end{array}\right]^{-i\left(\epsilon_{n}-\epsilon_{n^{\prime}}\right) \tau}
\end{gathered}
$$

To proceed further, we carry out a T-average for the quantity $\exp \left\{\frac{q_{x} e E T}{2 m^{*}} \tau\right\}$ over one period of radiation, $0=2 \pi \omega=$ $2 \pi \omega_{c}$. The polarization function is now written as

$$
\begin{aligned}
\Pi(\mathbf{q} \tau)=-2 & \sum_{n n^{\prime}}\left[n n^{\prime}\left(\begin{array}{ll}
x & y
\end{array}\right)\right]^{2} \frac{1}{\omega_{x} \tau}\left(i \omega_{x} \tau--i \omega_{x} \tau\right) \times \\
& -i \omega_{y} \tau\left[\begin{array}{l}
n-n^{\prime} \\
{ }^{\prime}
\end{array}{ }^{-i\left(\epsilon_{n}-\epsilon_{n^{\prime}}\right) \tau / \hbar}\right.
\end{aligned}
$$

where $\omega_{x}={ }_{x} \quad \pi(2 \quad), \omega_{y}=y \quad$. Now one can perform a Fourier transformation in $\tau$ to obtain

$$
\begin{gathered}
\Pi(\mathbf{q} \Omega)=-2 \sum_{n n^{\prime}}\left[n n^{\prime}\left(\begin{array}{cc}
x & y
\end{array}\right)\right]^{2} \frac{\left|n-n^{\prime}\right|}{\omega_{x}} \times \\
\ln \left[\frac{\omega_{p}^{2}-\left(\omega_{c}-\omega_{x}+\omega_{y}\right)^{2}}{\omega_{p}^{2}-\left(\omega_{c}+\omega_{x}+\omega_{y}\right)^{2}}\right]
\end{gathered}
$$

where $\omega_{n n^{\prime}}=\left|-{ }^{\prime}\right| \omega_{c}$.

The density-density correlation function can now also be calculated. Within the random phase approximation, the correlation function is given by

$$
(\mathbf{q} ; \omega)=\frac{\Pi(\mathbf{q} ; \omega)}{1+{ }_{q} \Pi(\mathbf{q} ; \omega)}
$$

Here $q=2 \pi^{2} \quad$ is the Coulomb interaction. The poles of $(\mathbf{q} ; \omega)$ are the modes of magnetoplasmons, $\omega_{p}$, given as

$$
\begin{aligned}
& 1=\frac{2 s}{\omega_{x}} \sum_{n m}\left[{ }_{n, n+m}\left(\begin{array}{ll}
x & y
\end{array}\right)\right]^{2}\left|{ }_{n}-{ }_{n+m}\right| \times \\
& \ln \left[\frac{\omega_{p}^{2}-\left(\omega_{c}-\omega_{x}+\omega_{y}\right)^{2}}{\omega_{p}^{2}-\left(\omega_{c}+\omega_{x}+\omega_{y}\right)^{2}}\right]
\end{aligned}
$$

In the above equation all energies are scaled by the zero field Fermi energy $\varepsilon_{F}$ and all wavenumbers are scaled by ${ }_{F}$; ${ }_{s}=* 2{ }_{F}$ is the plasma parameter. The principal plasmon mode has $=1$, and high terms are the Bernstein modes. The equation (21) can be easily evaluated for any value of electric and magnetic field. New features can also be observed in the imaginary part of the density-response function. The peaks originally at $\omega_{c}$ have now shifted and split into double peaks at $\omega_{c}+\omega_{y} \pm \omega_{x}$.

Assuming the principal mode is well separated from the higher modes, we obtain the analytical solution for $\omega_{p}$,

$\omega_{p}^{2}=\frac{\left(\omega_{c}+\omega_{x}+\omega_{y}\right)^{2} \exp \left(\begin{array}{lll}\omega_{x} & 2_{s} 1\end{array}\right)-\left(\omega_{c}-\omega_{x}+\omega_{y}\right)^{2}}{\exp \left(\begin{array}{llll}\omega_{x} 2_{s} & 1\end{array}\right)-1}$

where ${ }_{m}=\sum_{n}\left[{ }_{n, n+m}\left(\begin{array}{cc}x & y\end{array}\right)\right]^{2}\left|{ }_{n, k}-{ }_{n+m, k+q}\right|$. It can be shown easily that in the limit of $\rightarrow 0, \omega_{p}=\left(\omega_{q}^{2}(\quad)+\right.$

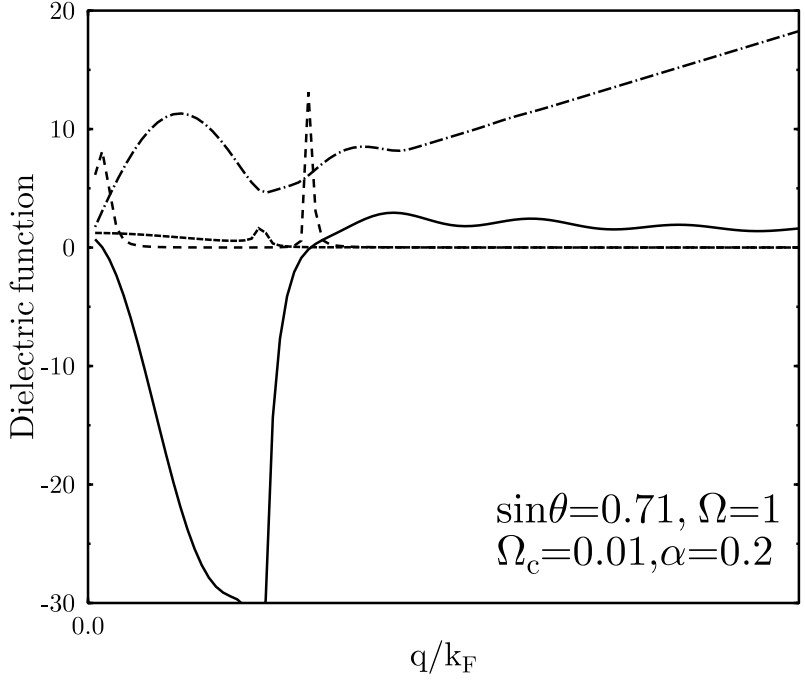

Fig. 1. Wave-vector-dependent dielectric functions, $s=1$. Solid line is the real part, the dotted line is the imaginary part and the dashed line is the absorption coefficient $\propto \Im(1)$. The dot-dashed line is the plasmon dispersion curve. The dimensionless quantities are $\Omega={ }^{-}{ }_{F}$ and $\Omega_{c}=$ c $F$.

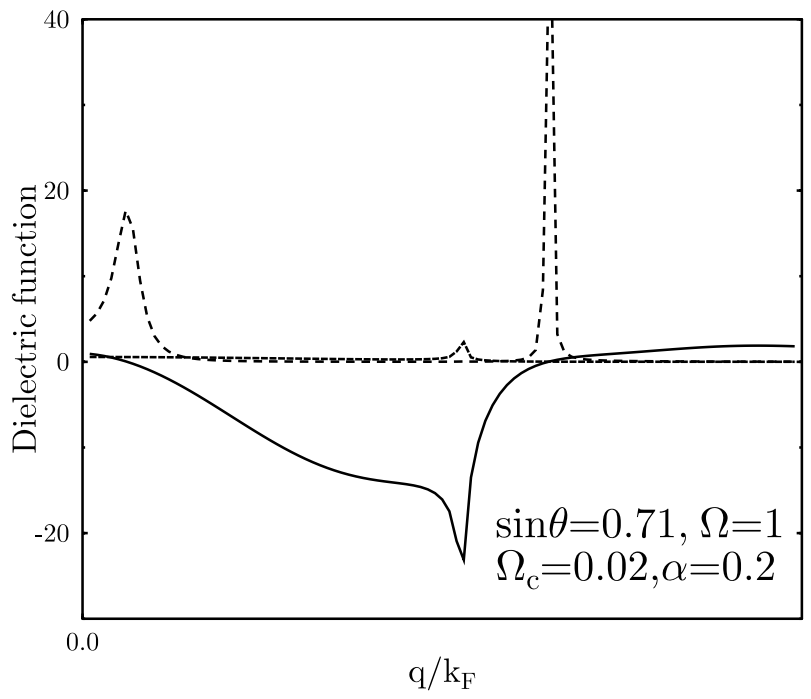

Fig. 2. Wave-vector-dependent dielectric functions, $s=1$. Solid line is the real part, the dotted line is the imaginary part and the dashed line is the absorption coefficient $\propto \Im(1)$.

$\left.\omega_{c}^{2}\right)^{1 / 2}$, where $\omega_{q}^{2}(\quad)=2 s \quad$ whose long-wavelength limit is $2 \pi r^{2} \quad *$. The dispersion and -dependence of $\omega_{p}$ for the principal mode are plotted in Figs. 1 and 2. We have used parameters of the GaAs semiconductor system: ${ }^{*}=$ $0067 e, s=10, \epsilon_{F}=11 \mathrm{meV}$ and $y=\sqrt{2}$. We also introduce a dimensionless field amplitude, = ${ }_{F}{ }^{*} \varepsilon_{F}^{2}$.

It can be seen that the electron-photon resonant coupling has qualitatively changed the property of dielectric response and magnetoplasmon. Figs. 1 and 2 show the wave-vectordependent dielectric function along the direction of ${ }_{x}=y$. The absorption coefficient is proportional to the structural function $\Im\left[\begin{array}{ll}1 & \epsilon\end{array}\right]$. The spectral weight of the absorption is 


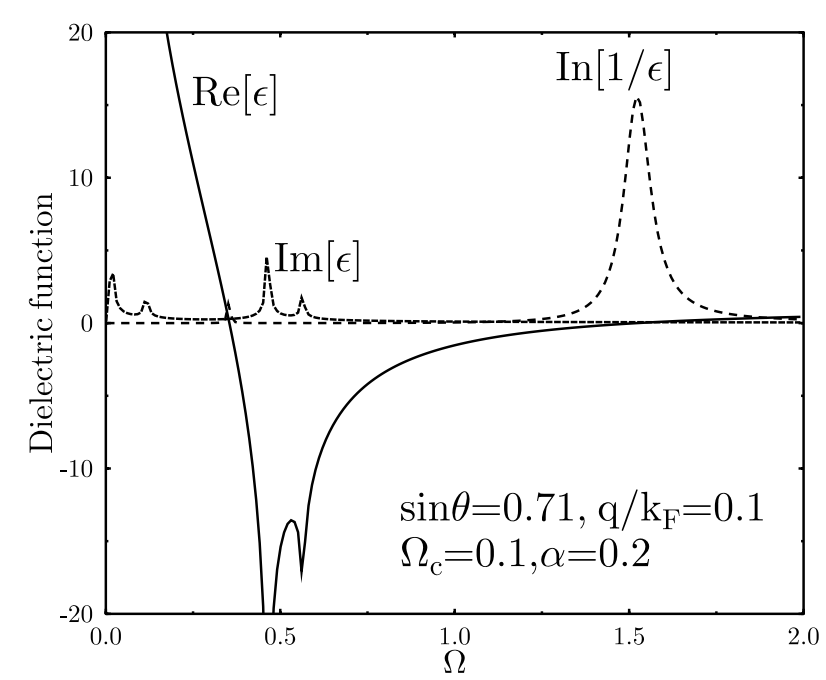

Fig. 3. Frequency-dependent dielectric functions and the absorption coefficient, $s=1$.

mainly located at two values, one at the principal plasmon (the sharp peak) and the other at the intralevel plasmon mode. As the magnetic field increases, the peaks will move to lower

(in weak regime)or a higher (in strong regime).

Fig. 3 depicts the frequency-dependent dielectric function and the light-scattering cross-section for small wavenumber,

$=01 F$. It can be seen that $\Re[\epsilon(\omega)]$ crosses zero twice, at around $\Omega \approx 035$ and $\Omega \approx 15$, indicating two possible collective modes (magnetopolariton modes). Close examination reveals that the mode at $\Omega \approx 035$ is heavily Landau damped due to a significant contribution of $\Im[\epsilon(\omega)]$ at this energy. The mode at $\Omega \approx 15$ is almost free of Landau damping. Therefore nearly all the spectral weight of lightscattering cross-section will be in the high-energy mode. This is shown by the behavior of $\Im[1 \epsilon(\omega)]$. In the absence of EM radiation, the high-energy mode carries all of the spectral weight. Due to the coupling with the radiation field, a very small amount of the spectral weight has been shifted to the low-energy mode. New features can also be observed in the imaginary part of the dielectric function. The peaks originally at $\omega_{c}$ have now shifted and split into double peaks at $\omega_{c}+\omega_{y} \pm \omega_{x}$.

In the absence of the radiation field, the behavior of $\omega_{p}(=$ $0)$ is well known. Under EM radiation of the cyclotron frequency, the plasma frequency increases with the radiation field, as shown in Fig. 4. The plasma frequency has a nearly linear dependence on the radiation field. The dependence of $\omega_{p}$ on the field is quite different in weak and strong radiation field regimes. At strong and intermediate radiation fields, $\omega_{p}$ increases with the inverse magnetic field. At weak radiation fields, $\omega_{p}$ increases slowly with .

The effect due to resonant coupling is most important in the finite- regime. The terms $\omega_{x}$ and $\omega_{y}$ are the dominant contributions in this regime. This leads to a sound-like (linear in ) magnetoplasmon mode in the large- regime. Such a dispersion curve is shown in Fig. 1 (dot-dashed line) together

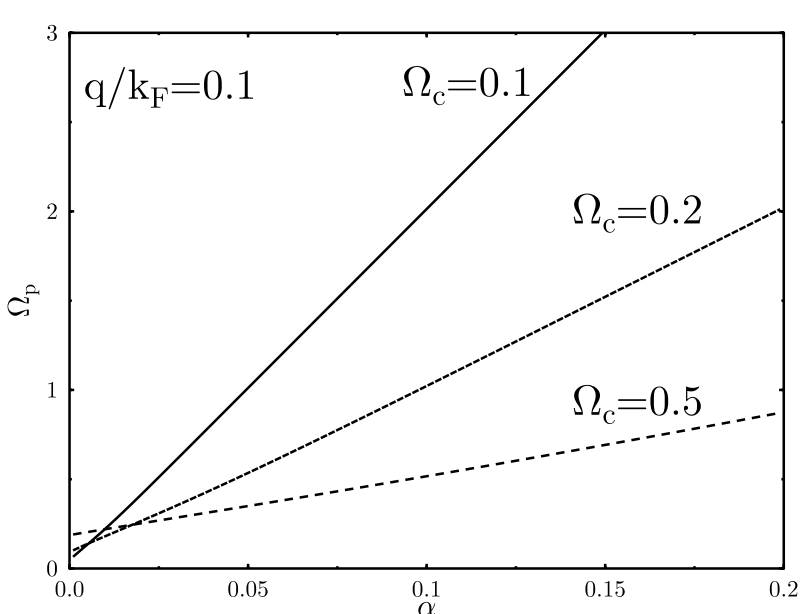

Fig. 4. Electric field dependent plasma frequency, $\Omega_{p}={ }^{-}{ }_{p} \quad F$

with the absorption rate. The speed of this sound-like wave is along the -direct and slightly higher along the direction. At low and high , the sound speed can be much higher than the Fermi velocity. The high value of $\omega_{p}$ also suggests that these modes suffers much less Laudau damping.

In conclusion, we have shown that when a radiation field whose frequency equals to the cyclotron frequency couples to an electronic system, the magnetopolariton mode increases with the radiation field and behaves like a sound wave in the large regime.

\section{ACKNOWLEDGMENT}

This work is supported in part by the Australian Research Council (Discovery Scheme) and the University of Wollongong (URG Scheme).

\section{REFERENCES}

[1] R. G. Mani, J. H. Smet, K. von Klitzing, V. Narayanamuri, W. B Johnson and V. Umansky, Nature 420, 646 (2002)

[2] M. A. Zudov, R. R. Du, L. N. Pfeiffer, and K. W. West, Phys. Rev. Lett. 90046807 (2003)

[3] P. D. Ye, W. Engel, D. C. Tsui, J. A. Simmons, J. R. Wendt, G. A Vawter, and J. L. Reno, Appl. Phys. Lett. 79, 2193 (2001)

[4] X. L. Lei and S. Y. Liu, Phys. Rev. Lett. 91, 226805 (2003)

[5] Adam C. Durst, Subir Sachdev, N. Read, and S.M. Girvin, Phys. Rev. Lett. 91, 086803 (2003)

[6] Junren Shi and X. C. Xie, Phys. Rev. Lett. 91, 086801 (2003)

[7] V. Ryzhii and V. Vyurkov, Phys. Rev. B68, 165406 (2003)

[8] J. C. Cao, Phys. Rev. Lett. 91, 237401 (2003); A. G. Markelz, N. G. Asmar, B. Brar, and E. G. Gwin, Appl. Phys. Lett. 69, 3975 (1996)

[9] W. Xu and C. Zhang, Appl. Phys. Lett. 68, 3305 (1996)

[10] W. Xu and C. Zhang, Phys. Rev. B55, 5259 (1997)

[11] C. Zhang, Phys. Rev. B66, 081105 (2002)

[12] N. G. Asmar, A. G. Markelz, E. G. Gwinn, J. Cerne, M. S. Sherwin, K. L. Campman, P. E. Hopkins, and A. C. Gossard, Phys. Rev. B51, 1804 (1995)

[13] A. G. Markelz, N. G. Asmar, B. Brar, and E. G. Gwin, Appl. Phys. Lett 69, 3975 (1996)

[14] B. N. Murdin, W. Heiss, C. J. G. M. Langerak, S.-C. Lee, I. Galbraith, G. Strasser, E. Gornik, M. Helm, and C. R. Pidgeon, Phys. Rev. B55 5171 (1997)

[15] C. J. G. M. Langerak, B. N. Murdin, B. E. Cole, J. M. Chamberlain, M. Henini, M. Pate, G. Hill, Appl. Phys. Lett. 673453 (1995)

[16] C. Zhang, Appl. Phys. Lett. (2001) 